\title{
Conjugate Gradient Approach for Linear Optimal Control of Damped Harmonic Oscillator
}

\author{
Sie Long Kek ${ }^{1}$, Sy Yi Sim², Wah June Leong ${ }^{3}$, Chuei Yee Chen ${ }^{4}$ \\ ${ }^{1,2}$ Universiti Tun Hussein Onn Malaysia \\ 84600 Pagoh, Muar, Malaysia \\ 11slkek@uthm.edu.my; 2sysim@uthm.edu,my \\ ${ }^{3,4}$ Universiti Putra Malaysia \\ 43400 Seri Kembangan, Selangor, Malaysia \\ ${ }^{3}$ leongwj@upm.edu.my; ${ }^{4}$ cychen@upm.edu.my
}

\begin{abstract}
In this paper, applying the conjugate gradient method to solve the linear optimal control problem is discussed. In the optimization theory, the conjugate gradient method is an efficient computational approach for solving the unconstrained optimization problem, specifically, for quadratic case. Since the linear optimal control problem consists of the quadratic cost function and the linear dynamical system, the practical application of the conjugate gradient method to this kind of problem would be addressed. In our study, the necessary conditions for optimality for the linear optimal control problem are highlighted. Then, the equivalent optimization problem is formulated and the gradient function, which is given by the stationary condition, is evaluated. On this basis, the search direction, which satisfies the conjugacy, is determined definitely. During the iterative procedure, the control sequence is calculated such that the state sequence could be obtained. Once the convergence is achieved, the optimal solution of the linear optimal control problem is obtained. For illustration, the optimal control of damped harmonic oscillator is discussed. The results obtained show the efficiency of the approach used. In conclusion, the application of the conjugate gradient method to linear optimal control problem of the damped harmonic oscillator is highly presented.
\end{abstract}

Keywords: Conjugate Gradient Method, Linear Optimal Control, Necessary Conditions, Iterative Procedure, Damped Harmonic Oscillator

\section{Introduction}

Optimal control is a branch of applied mathematics and control engineering. The applications of the optimal control, which cover the real world applications, have been well-defined in the literature [1], [2]. Both linear and nonlinear models in the optimal control problem attract the interest of researchers in optimizing and controlling the dynamical system [3]. The linear optimal control, which is known as the linear quadratic regulator, is a standard calculation procedure, and the existing of the optimal solution is guaranteed definitely [4], [5]. However, the use of the linear optimal control and the corresponding optimal solution are still providing a potential exploration in the real practical applications.

In this paper, the conjugate gradient method, which is an efficient technique [6] for solving the unconstraint optimization problem, is proposed to handle the linear optimal control problem. By virtue of this, the Hamiltonian function is defined, and the corresponding first-order necessary conditions are derived. It is important to emphasize that the stationary condition, which is referred to generate the optimal feedback law, represents the gradient function as an equivalent optimization problem is defined [7], [8]. On this basis, the search direction is determined and the conjugacy property is ensured [9]. During the iterative procedure, the control sequence is updated through the line search calculation, in turn, to produce the optimal state trajectory [10]. Once the convergence is achieved, the optimal solution of the linear optimal control problem is obtained. For illustration, the optimal control of damped harmonic oscillator is investigated. As a result, the efficiency of the approach used is highly demonstrated.

The paper is organized as follows. In Section 2, the problem description on the linear optimal control is given. In Section 3 , the conjugate gradient approach is discussed. From the necessary conditions of the linear optimal control problem, the stationary condition represents the gradient function for an equivalent optimization problem formulated. In Section 4 , the illustration on the optimal control of a damped harmonic oscillator is studied. Finally, some concluding remarks are made. 


\section{Problem Description}

Consider the following linear optimal control problem [2], [3],

$$
\min _{u} J(u)=\frac{1}{2} x(N)^{\mathrm{T}} S(N) x(N)+\frac{1}{2} \sum_{k=0}^{N-1}\left(x(k)^{\mathrm{T}} Q x(k)+u(k)^{\mathrm{T}} R u(k)\right)
$$

subject to

where $u(k) \in \mathfrak{R}^{m}, k=0,1, \cdots, N-1$, and $x(k) \in \mathfrak{R}^{n}, k=0,1, \cdots, N$, are, respectively, the control sequences and the state sequences, whereas $A$ is an $n \times n$ transition matrix, $B$ is an $n \times m$ control coefficient matrix, $S(N)$ and $Q$ are $n \times n$ positive semi-definite matrices, and $R$ is a $m \times m$ positive definite matrix. Here, $J$ is the scalar cost function and $x_{0} \in \mathfrak{R}^{n}$ is the initial state.

This optimal control problem, which is referred to as Problem $(\mathrm{P})$, is a discrete-time linear quadratic regulator problem with the linear dynamical system and the quadratic criterion cost function [3], [4]. Solving this optimal control problem involves solving the two-point boundary-value (TPBV) problem, where the calculation procedure is complicated to obtain the optimal solution [5]. Moreover, determining the optimal control law requires more computational efforts on computing the Riccati solution and the feedback gain in which the feedback state control could be obtained in linear of the state variable. By virtue of this, in this paper, we suggest to apply the conjugate gradient approach to solve this optimal control problem.

\section{Conjugate Gradient Computation Approach}

Now, let us define the Hamiltonian function as follows,

$$
H(k)=\frac{1}{2}\left(x^{\mathrm{T}}(k) Q x(k)+u^{\mathrm{T}}(k) R u(k)\right)+p^{\mathrm{T}}(k+1)(A x(k)+B u(k)) .
$$

Then, the augmented cost function becomes

$$
J^{\prime}=\frac{1}{2} x^{\mathrm{T}}(N) S(N) x(N)+\sum_{k=0}^{N-1}\left(H(k)-p^{\mathrm{T}}(k+1) x(k+1)\right) .
$$

By considering the initial and terminal conditions for the costate, the augmented cost function given by (3) can be rewritten as

$$
J^{\prime}=\frac{1}{2} x^{\mathrm{T}}(N) S(N) x(N)+p^{\mathrm{T}}(0) x(0)-p^{\mathrm{T}}(N) x(N)+\sum_{k=0}^{N-1}\left(H(k)-p^{\mathrm{T}}(k) x(k)\right) .
$$

Applying the concept of the calculus of variation [4], [5], the necessary conditions for optimality are obtained as follows:

(a) Stationary condition:

$$
0=\frac{\partial H(k)}{\partial u(k)}=R u(k)+B^{\mathrm{T}} p(k+1)
$$


(b) Co-state equation:

$$
p(k)=\frac{\partial H(k)}{\partial x(k)}=Q x(k)+A^{\mathrm{T}} p(k+1)
$$

(c) State equation:

$$
x(k+1)=\frac{\partial H(k)}{\partial p(k+1)}=A x(k)+B u(k)
$$

with boundary values

$$
x(0)=x_{0} \text { and } p(N)=S(N) x(N) .
$$

Define the discrete-time linear optimal control problem given by (1) as an optimization problem, which is referred to as Problem (Q), as follows:

$$
\text { Minimize } J(u) \text { subject to } u=[u(1), u(2), \cdots, u(N-1)] \text {. }
$$

Suppose the gradient function $g$ is defined by

$$
g\left(u^{(i)}\right)=\nabla_{u} H(k)
$$

which is given by (5). For arbitrary initial control $u^{(0)} \in \mathfrak{R}^{m}$, the initial gradient $g^{(0)}$ and the initial direction $d^{(0)}$ are given by

$$
g^{(0)}=g\left(u^{(0)}\right) \text { and } d^{(0)}=-g^{(0)} .
$$

To update the control sequences, applying the following linear search equation [6], [7],

$$
u^{(i+1)}=u^{(i)}+\alpha_{i} \cdot d^{(i)}
$$

where $\alpha_{i} \in \mathfrak{R}$ is the step size and its value can be calculated by solving the one-dimensional minimization to give

$$
\alpha_{i}=\underset{\alpha \geq 0}{\arg \min } J\left(u^{(i)}+\alpha \cdot d^{(i)}\right)
$$

After that the gradient $g$ and the direction $d$ are updated, respectively, by

$$
g^{(i+1)}=g\left(u^{(i+1)}\right)
$$

and

$$
d^{(i+1)}=-g^{(i+1)}+\beta_{i} d^{(i)}
$$

with the step-size $\beta$ given by 


$$
\beta_{i}=\frac{g^{(i+1) \mathrm{T}} Q d^{(i)}}{d^{(i) \mathrm{T}} Q d^{(i)}}
$$

for $i=0,1,2, \ldots$ represents the iteration numbers.

From the discussion above, we summarize the calculation procedure as an iterative algorithm as follows.

Iterative Algorithm

Data Choose the arbitrary initial control $u^{(0)}$. Compute the initial gradient $g^{(0)}$ and the initial direction $d^{(0)}$ from (11). Set $i=0$.

Step 1 Solve the state equation (7) forward in time from $k=0$ to $k=N$ with the initial condition $x(0)=x_{0}$ to obtain $x(k)^{i}$, $k=0,1, \cdots, N$.

Step 2 Solve the costate equation (6) backward in time from $k=N$ to $k=0$ with the boundary condition $p(N)=S(N) x(N)$, where $p(k)^{i}$ is the solution obtained.

Step 3 Calculate the value of the cost functional $J\left(u^{(i)}\right)$ from (1).

Step 4 Determine the step size $\alpha_{i}$ from (13).

Step 5 Update the control $u^{(i+1)}$, the gradient $g^{(i+1)}$, and the direction $d^{(i+1)}$, respectively, from (12), (14) and (15) with $\beta_{i}$ from (16). If the gradient $g^{(i+1)}=0$, stop, else set $i=i+1$, go to Step 1 .

\section{Remarks:}

(a) The gradient of Problem $(\mathrm{Q}), \nabla_{u} J(u)$ is equivalent to the gradient of Problem $(\mathrm{P}), \nabla_{u} H(k)$. Once the convergence is reached, $\nabla_{u} J(u)=\nabla_{u} H(k)$ and the stationary condition (5) is satisfied.

(b) In Steps 1 and 2, the state sequences and the costate sequences are calculated to ensure the necessary conditions (7) and (6) are satisfied.

(c) The control sequences are computed in such a way that the open-loop control sequences could be generated tractably.

(d) By using the approach proposed, it is verified that the conjugacy is satisfied.

\section{Illustrative Example}

Consider the system dynamic of a damped harmonic oscillator [5], given by

$$
\ddot{x}+2 \delta \omega \dot{x}+\omega^{2} x=u
$$

which can be written in the state equation form as

$$
\dot{x}=\left(\begin{array}{cc}
0 & 1 \\
-\omega^{2} & -2 \delta \omega
\end{array}\right) x+\left(\begin{array}{l}
0 \\
1
\end{array}\right) u
$$

with the natural frequency $\omega$ and damping ratio $\delta$. Taking the discretization on the system dynamics with the sampling time $\Delta t$, the state equation becomes

$$
x(k+1)=A_{d} x(k)+B_{d} u(k)
$$

where $A_{d}=e^{A \cdot \Delta t}$ and $B_{d}=\left(e^{A \cdot \Delta t}-1\right) \cdot A^{-1} \cdot B$. By setting the sampling time $\Delta t=0.65$ seconds, the natural frequency $\omega=0.8$ and the damping ratio $\delta=0.1$, the discrete-time state equation is written by 


$$
x(k+1)=\left(\begin{array}{cc}
0.87226 & 0.58990 \\
-0.37754 & 0.77788
\end{array}\right) x(k)+\left(\begin{array}{c}
0.19959 \\
2.15240
\end{array}\right) u(k)
$$

for $k=0,1,2, \ldots, 19$. Let us associate this state equation with the performance index defined by

$$
J(u)=\frac{1}{2} x(N)^{\mathrm{T}} S(N) x(N)+\frac{1}{2} \sum_{k=0}^{N-1}\left(x(k)^{\mathrm{T}} Q x(k)+u(k)^{\mathrm{T}} R u(k)\right)
$$

where $S(N), Q$ and $R$ are the weighting matrices. Here, the aim is to determine the optimal control law $u(k)$ such that the performance index $J$ is minimized over the state equation with the initial state $x(0)=x_{0}$.

This problem, which is referred to as Problem (P), is the linear optimal control problem of damped harmonic oscillator.

Now, using the approach discussed in Section 3 to solve Problem $(\mathrm{P})$, where the weighting matrices and the initial state are, respectively, given by

$$
S(N)=I_{2 \times 2} ; Q=0.1 I_{2 \times 2} ; R=1 ; N=20 ; x_{0}=(1010)^{\mathrm{T}} .
$$

The GNU Octave 4.4.0 is used to implement the approach proposed. When the iteration is terminated, the simulation result, which is shown in Table 1, is obtained. The trajectories of control, state and costate are shown, respectively, in Figures 1,2 , and 3. The optimal control regulates the solution of the damped harmonic oscillator tends to zero and assists the system dynamics reach to a stable behaviour. It is claimed that the optimal solution is obtained once the stationary condition, which is shown in Table 4, is satisfied numerically.

Table 1: Simulation result

\begin{tabular}{|c|c|c|}
\hline Number of Iterations & Optimal Cost & Elapsed time (s) \\
\hline 133 & 39.898782 & 4.27056 \\
\hline
\end{tabular}

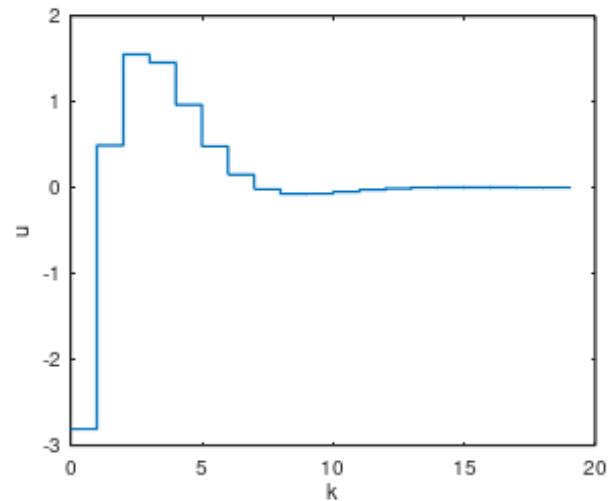

Fig 1: Trajectory of control

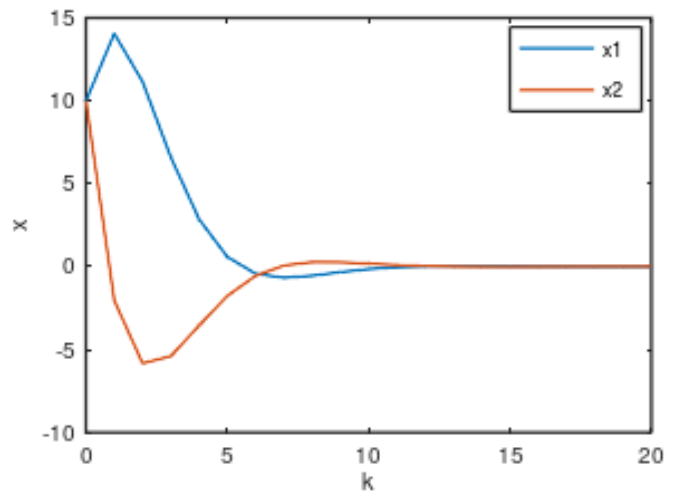

Fig. 2: Trajectory of state 


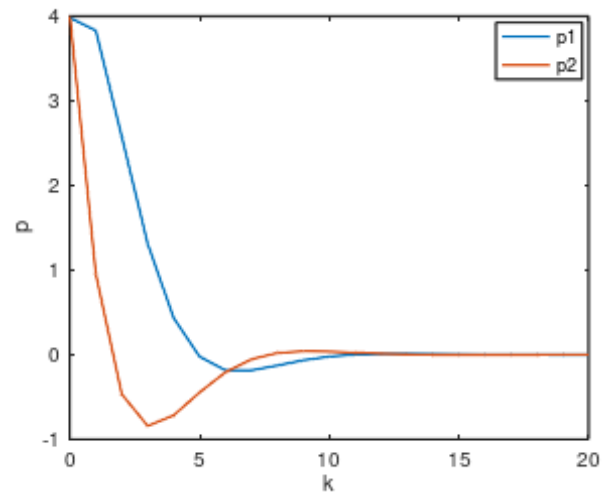

Fig. 3: Trajectory of costate

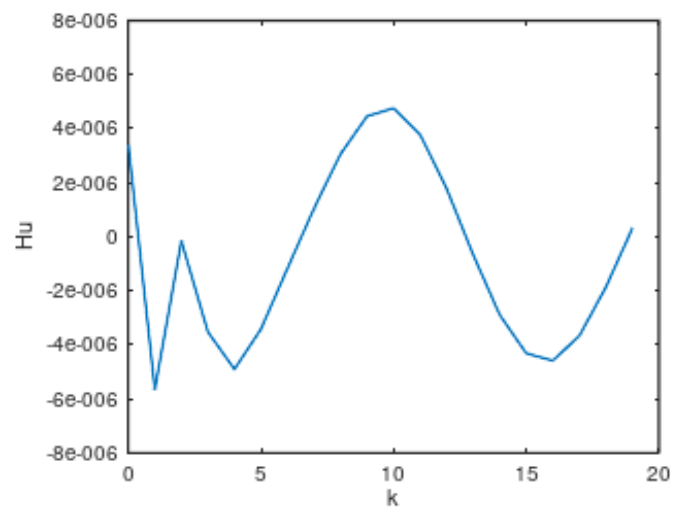

Fig. 4: Stationary condition

\section{Concluding Remarks}

The use of the conjugate gradient method in solving the linear optimal control problem was discussed in this paper. The first-order necessary conditions for the linear optimal control problem were derived, and the equivalent optimization problem was formulated. Here, the gradient function, which is represented by the stationary condition, was evaluated. By using the line search calculation, the search direction was generated and the conjugacy was determined. The optimal control of the damped harmonic oscillator was demonstrated, and the results obtained showed the efficiency of the approach proposed. In conclusion, the applicability of the approach proposed for solving the linear optimal control problem is highly presented.

\section{Acknowledgements}

The authors would like to acknowledge the Universiti Tun Hussein Onn Malaysia (UTHM) and the Ministry of Higher Education (MOHE) for the financial support for this study under the research grant FRGS 2018-1, VOT K079.

\section{References}

[1] R.E. Kalman, "Contributions to the theory of optimal control," Boletın de la Sociedad Matematica Mexicana, vol. 5, pp. 102-119, 1960.

[2] A.E. Bryson and Y.C. Ho, Applied Optimal Control. Hemisphere, Washington, DC, USA, 1975.

[3] K.L. Teo, C.J. Goh and K.H. Wong, A Unified Computational Approach to Optimal Control Problems. New York: Longman Scientific \& Technical, 1991.

[4] D.E. Kirk, Optimal Control Theory: An Introduction. Mineola, NY: Dover Publications, New York, 2004.

[5] F.L. Lewis, V. Vrabie and V.L. Symos, Optimal Control. $3^{\text {rd }}$ Ed. John Wiley \& Sons, Inc., New York, 2012.

[6] E.K.P. Chong, and S.H. Zak, An Introduction to Optimization. $4^{\text {th }}$ Ed. John Wiley \& Sons, Inc., Hoboken, New Jersey 2013.

[7] L.S. Lasdon and S.K. Mitter, "The conjugate gradient method for optimal control problems," IEEE Transactions on Automatic Control, vol. 12, pp. 132-138, 1967.

[8] S.L. Kek, W.J. Leong, S.Y. Sim and K.L. Teo, "Application of conjugate gradient approach for nonlinear optimal control problem with model-reality differences," Applied Mathematics, vol. 9, no. 8, pp. 940-953, 2018.

[9] S.L. Kek, M.I. Abd Aziz and K.L. Teo, "A gradient algorithm for optimal control problems with model-reality differences," Numerical Algebra, Control and Optimization, vol. 5, Issue 3, 252-266, 2015.

[10] E. Nwaeze, "An extended conjugate gradient method for optimizing continuous-time optimal control problems," Canadian Journal on Computing in Mathematics, Natural Sciences, Engineering \& Medicine, vol. 2, pp. 39-44, 2011. 